\title{
Instrumentation for beam radiation and luminosity measurement in the CMS experiment using novel detector technologies
}

\author{
Moritz Guthoff, On behalf of the CMS collaboration \\ CERN, CH-1211 Geneve 23, Switzerland
}

\section{A R T I C L E I N F O}

\section{Keywords:}

CMS

Beam instrumentation

Luminosity

Machine induced background

\begin{abstract}
A B S T R A C T
The higher energy and luminosity of the LHC initiated the development of dedicated technologies for radiation monitoring and luminosity measurement. A dedicated pixelated luminosity detector measures coincidences in several three-layer telescopes of silicon pixel detectors to arrive at a luminosity for each colliding LHC bunch pair. In addition, charged particle tracking allows to monitor the location of the collision point. The upgraded fast beam conditions monitor measures the particle flux using 24 two-pad single crystalline diamond sensors, equipped with a fast front-end ASIC produced in $130 \mathrm{~nm}$ CMOS technology. The excellent time resolution is used to separate collision products from machine induced background. A new beam-halo monitor at larger radius exploits Cherenkov light produced by relativistic charged particles in fuzed quartz crystals to provide direction sensitivity and time resolution to separate incoming and outgoing particles. The back-end electronics of the beam monitoring systems includes dedicated modules with high bandwidth digitizers developed in both VME and microTCA standards for per bunch beam measurements and gain monitoring. All new and upgraded sub-detectors have been taking data from the first day of LHC operation in April 2015. Results on their commissioning and essential characteristics using data since the start-up of LHC will be presented.
\end{abstract}

\section{Introduction}

The Beam Radiation Instrumentation and Luminosity (BRIL) project of the CMS experiment is responsible for measuring: luminosity, machine induced background (MIB) [1] and beam timing. BRIL systems also provide active protection in case of intense beam loss events [2] and give various inputs to the CMS trigger system. To achieve these goals a variety of detectors, operated outside of the central CMS data acquisition, are used. All detectors must be functional whenever there is beam in the LHC.

Additionally radiation simulations of the CMS detector [3,4] using FLUKA [5,6] and MARS [7] are performed. Example applications are: detector hit rates, radiation damage, activation of material. Protonproton and heavy-ion collisions can be simulated, as well as machine induced background simulations following a simulation of the LHC performance and the particle showers inside the LHC tunnel [8].

\section{Detector systems and technologies}

The location of the various BRIL systems inside CMS is shown in Fig. 1.

\subsection{Pixel Luminosity Telescope (PLT)}

The PLT [9] consists of 16 small-angle particle tracking telescopes placed at $Z= \pm 1.8 \mathrm{~m}$ from the interaction point at a radius of about
$5 \mathrm{~cm}$. Each telescope consists of 3 planes of hybrid silicon pixel detectors developed for the phase-0 CMS Pixel detector. The detector backend readout has been adopted from the Pixel system. A fast cluster counting signal (Fast-OR) registers if a sensor plane has a hit on a $25 \mathrm{~ns}$ basis. Threefold coincidences of the fast-OR output are used to count luminosity particles as function of the bunch crossing (BX). A $4 \times 4 \mathrm{~mm}^{2}$ mask is applied to the center plane to remove edge effects and reduce the acceptance of accidental coincidences. The position sensitive pixel readout, operating at a trigger frequency of $2 \mathrm{kHz}$, is used to record tracking information to correct for systematic detector effects. Occupancy histograms for all planes of one telescope are shown in Fig. 2. The mask shadows on the outer planes show the imperfection of the alignment, which is, however, fully sufficient. From the reconstructed tracks in the PLT the size and position of the beam spot can be computed.

\subsection{Fast beam conditions monitor (BCM1F)}

The BCM1F detector uses 24 single-crystalline diamond detectors with two-pad metallization. A dedicated fast ASIC pre-amplifier shows particle hits with $\sim 10 \mathrm{~ns}$ FWHM. A picture of the sensors and the cshaped detector PCB is shown in Fig. 3. The detectors are placed at the strategic location of $Z= \pm 1.8 \mathrm{~m}$, where incoming machine induced background particles and outgoing luminosity products are separated in time by $12.5 \mathrm{~ns}$ (alternating between background and collisions in $25 \mathrm{~ns}$ operation) to allow to discriminate between the two sources via 


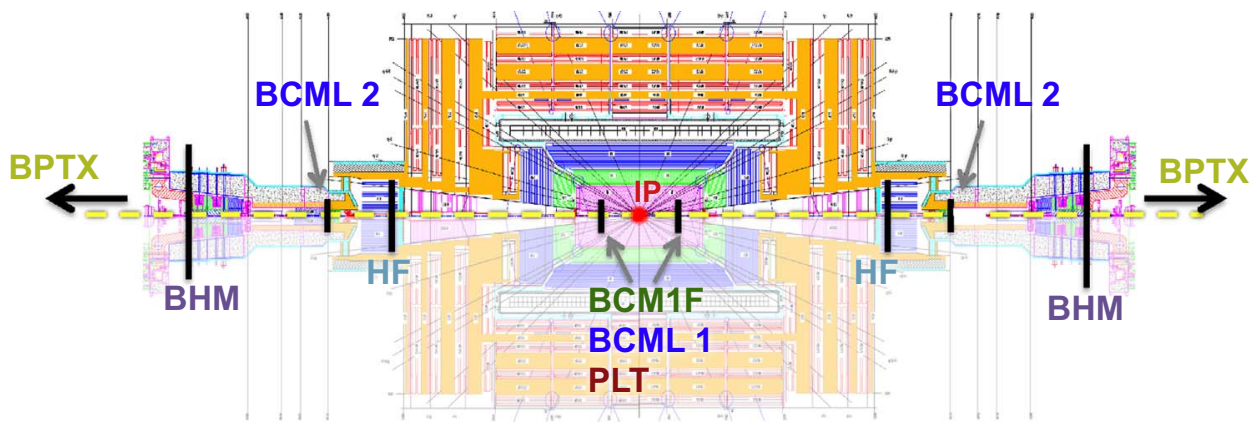

Fig. 1. Position of the various BRIL systems inside CMS.

timing. An offline analysis of the timing structure (Fig. 4) shows the two peaks corresponding to machine induced background and collision products. The signals are discriminated and histogrammed as a function of time with 4 bins per bunch crossing in a dedicated Realtime Histogramming Unit (RHU).

A $\mu$ TCA based readout system is used to process the analog pulses [10]. A modified FMC125 ADC board with $1.25 \mathrm{GS} / \mathrm{s}$ digitizes the pulse information. The GLIB carrier board is equipped with a Xilinx Virtex-6 FPGA. A peak finding algorithm measures the peak height and timing with respect to the LHC orbit clock. Both values are histogrammed in a dead-time free way in the FPGA. Advanced peak filtering will be implemented to mitigate pile-up.

\subsection{Beam halo monitor (BHM)}

The BHM system [11] measures muons created in beam loss events, so-called muon halo. Directional Cherenkov light is created in quartz bars and photomultiplier tubes are used for light detection, all being placed inside an iron tube for magnetic shielding. Fig. 5 shows the difference in signal strength between forward and backward directed particles. 20 detectors per beam are placed pointing towards the LHC tunnel. Fast timing allows additional separation of MIB hits from collision products. Fig. 6 shows the response of BHM to a non-colliding bunch train with MIB hits only, and a colliding bunch train where the MIB is overlayed with random hits from collisions arriving 6 BX later.

\subsection{Hadron forward calorimeter for luminosity (HFlumi)}

The hadron forward calorimeter (HF) of CMS includes a secondary readout chain used for Luminosity measurements. Detector hits are histogrammed as a function of bunch crossing. Triple buffering enables dead-time free readout independent of the CMS DAQ.

\subsection{Beam condition monitor for loss monitoring (BCML)}

This system is part of the LHC Beam Loss Monitoring system , using $1 \times 1 \mathrm{~cm}^{2}, 400 \mu \mathrm{m}$ thick, poly-crystalline diamond sensors instead of ionization chambers of the LHC. Since the detector current is measured directly, the low dark current of the diamond sensors is an important feature. If the current in one of the 16 channels exceeds the pre-defined abort threshold, the LHC beams are automatically dumped.

\subsection{Beam position for timing at the experiments (BPTX)}

This system uses LHC beam position monitor detectors to measure the exact timing of the incoming bunches. The detectors are placed $175 \mathrm{~m}$ away from the interaction point. The signals are analyzed together with the orbit clocks in an oscilloscope to compute the time difference between the two beams, $\Delta T(\mathrm{~B} 1-\mathrm{B} 2)$, and phases between beams and CMS clock. The signals are also discriminated and sent to a logic unit to compute various technical trigger signals. Measurement of bunch intensity is also possible and will be done in the future.

\section{Detector performance and measurements}

\subsection{Luminosity}

Measuring the luminosity precisely is necessary for all measurements of cross-sections at CMS. The BRIL systems used for luminosity measurements are: PLT, BCM1F and HFlumi. An online luminosity value is reported every $\sim 1.4 \mathrm{~s}\left(2 \times 10^{14}\right.$ LHC turns $)$, including bunchby-bunch values, visualized in the CMS and LHC control rooms. The online luminosity is used by the LHC to optimize the beam overlap and increase the collision rate, and bunch-by-bunch values are used to
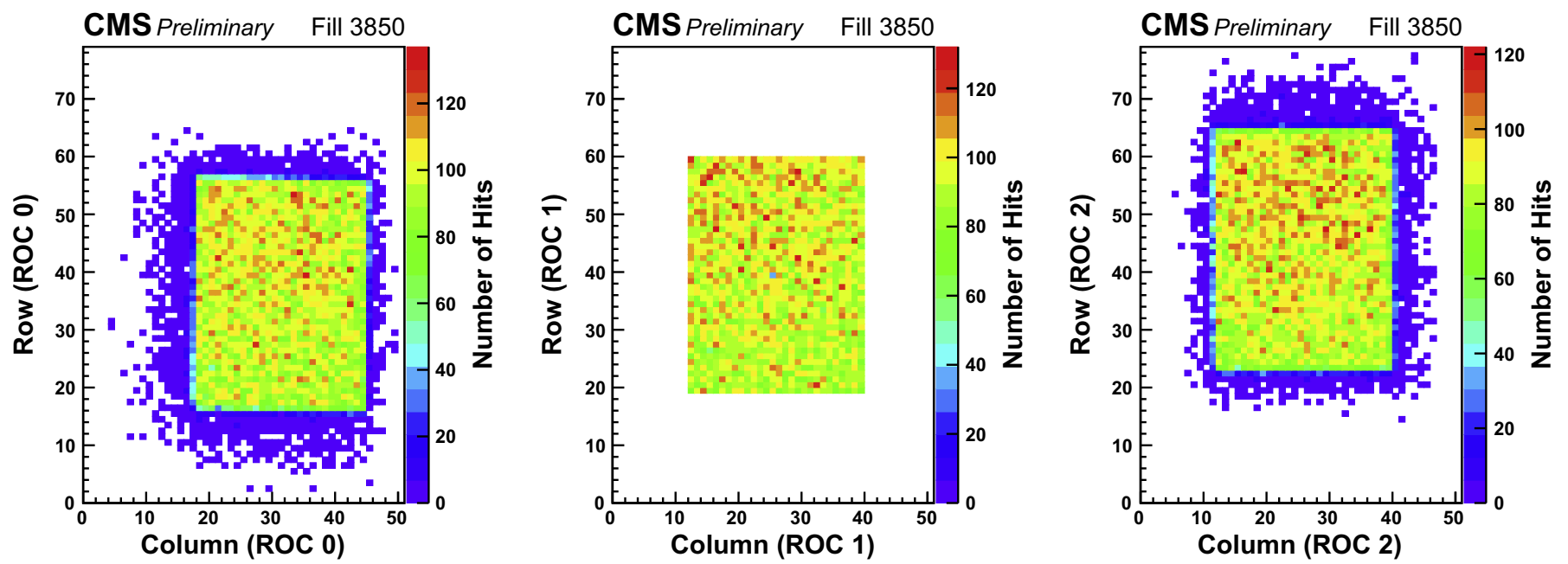

Fig. 2. Occupancy histograms of each plane of one PLT telescope for one example fill. 


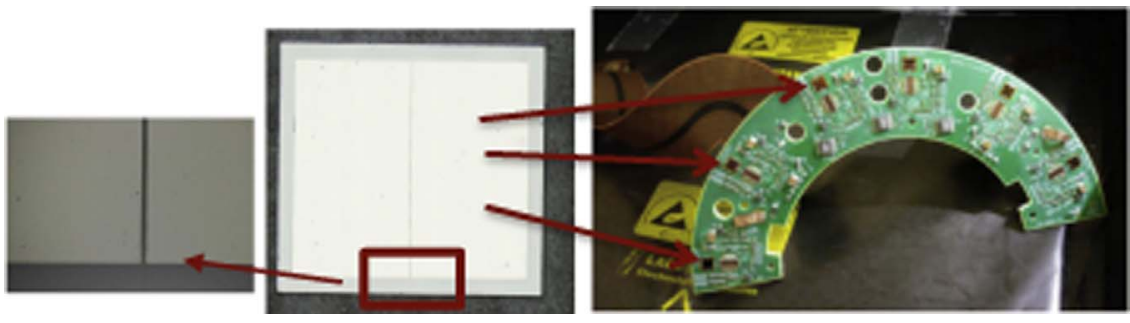

Fig. 3. Picture of the BCM1F C-shaped PCB and the split-pad metallized diamond sensors.

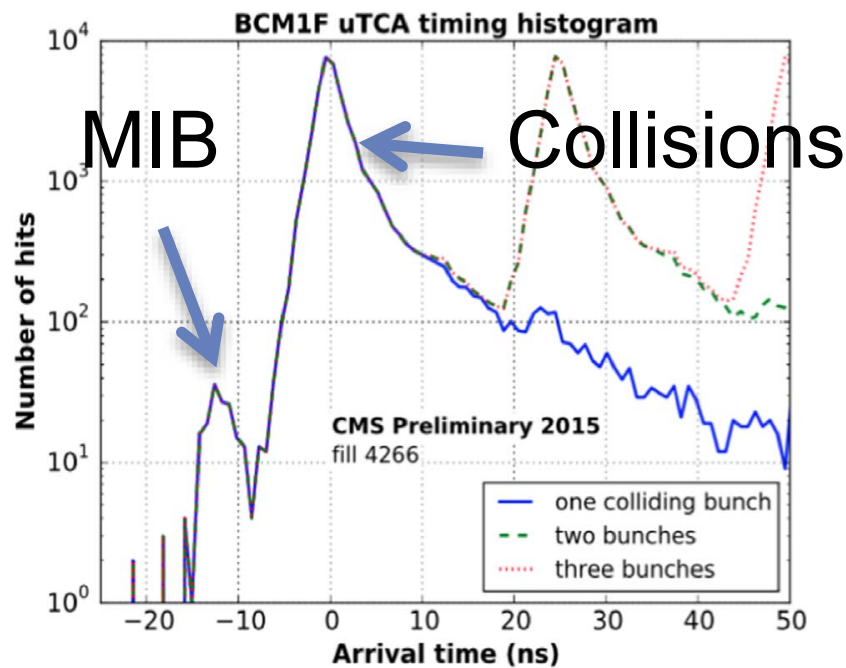

Fig. 4. Timing of the BCM1F detector hits obtained from offline processing of the raw data obtained with the $\mu \mathrm{TCA}$ system.

compute the emittance of the beam. For offline analyses, all luminosity values are stored in a database allowing CMS collaborators to download luminosity information and apply the latest offline calibration constants. The absolute calibration of a luminometer is performed in a Van der Meer (VdM) scan, where the absolute luminosity can be calculated, if the width of the beam overlap and the beam intensity is known, and put into relation with the detector readings. To measure the width of the beam overlap, both beams are offset and scanned across. The detector rate as function of beam separation is parameterized to compute the visible cross-section of this detector, correlating measured detector hit rate with absolute Luminosity. The visible cross-section is calculated by [13]

$\sigma_{v i s}=\frac{2 \pi \Sigma_{X} \Sigma_{Y} R}{N_{1} N_{2} f}$

with $N$ : bunch intensity, $f$ : accelerator revolution frequency, $R$ : measured rate at maximum beam overlap and $\Sigma$ : width of the beam overlap. The analysis is performed on a bunch-by-bunch level.

$\Sigma$ is taken from a double gaussian fit to the data, an example is shown in Fig. 7.

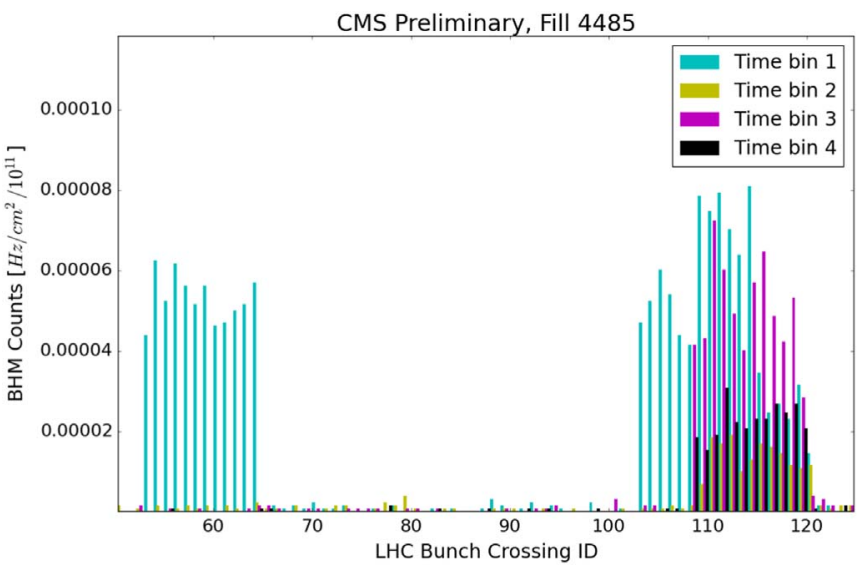

Fig. 6. Timing histogram obtained with BHM showing a non-colliding and a colliding bunch train.

\subsection{Machine induced background (MIB)}

Machine induced background (MIB) particles are several orders of magnitude rarer than collision products. A measurement of this background is desirable since an increase of such background is an indication for bad beam quality that may affect data-taking performance. The systems used for machine induced background measurements are $\mathrm{BCM} 1 \mathrm{~F}, \mathrm{BCML}$ and $\mathrm{BHM}$. The $\mathrm{BCM} 1 \mathrm{~F}$ is placed at low radii and most sensitive to MIB originating from local beam gas interactions. BCM1F background measurements are well correlated with the local vacuum pressure [1]. Fig. 8 shows how the background measurement follows changes in the vacuum pressure. The vacuum pressure is correlated with luminosity due to outgassing caused by collision products. As the BHM detector measures long ranging muons, it is more sensitive to MIB originating further upstream in the LHC. In the muon halo, MIB particles originating from beam halo hitting the tertiary collimators are more dominant. Fig. 9 shows the signals measured in BHM during a collimator scan, where the tertiary collimators close to CMS are moved in and a beam loss is created. BCM1F and BHM give complementary information, allowing to study the nature of beam loss events, and separate contributions from beam gas and beam halo interactions. The BCML system uses different integrating windows that allow to study the timing structure of fast

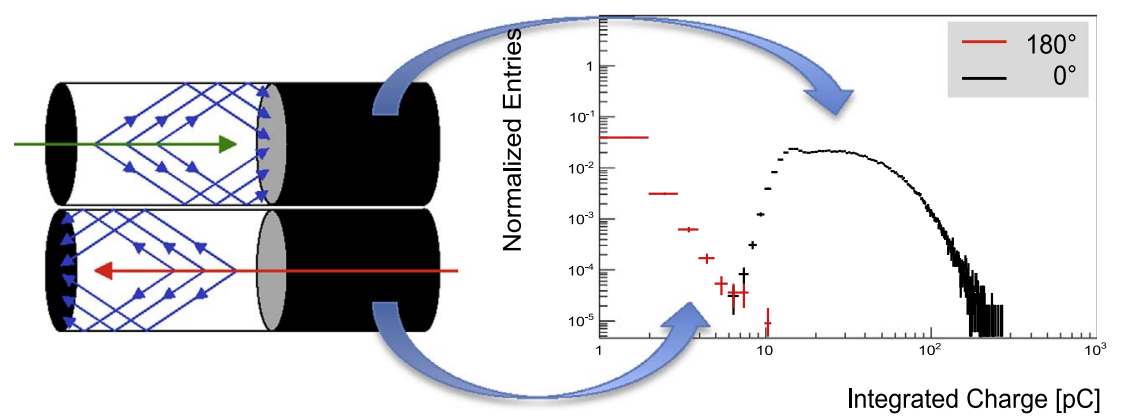

Fig. 5. Directionality of the BHM detectors. Forward particles give high amounts of signal, while backwards directed particles are suppressed [11]. 

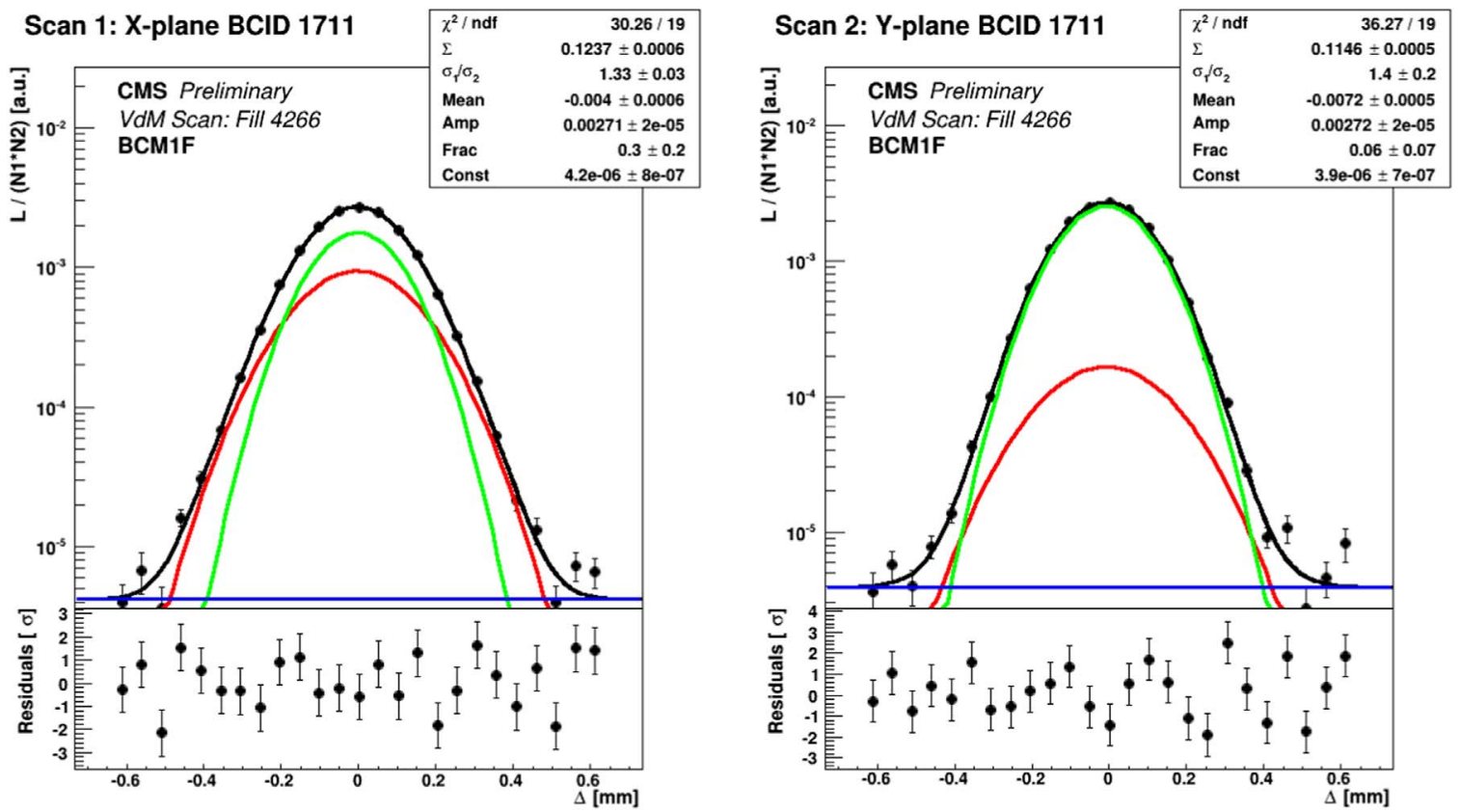

Fig. 7. Double gaussian fit to BCM1F data obtained in a VdM fill.

beam loss events (duration $<1$ s) like UFOs [2].

\subsection{Active protection}

Active protection of the silicon detectors inside CMS is achieved by the BCML system. The data acquired by the system is used for checks of functionality and stability. Most important for this system is an availability of $100 \%$. Radiation induced signal degradation was observed during Run 1 [12], due to the relatively low operational HV. Increased $\mathrm{HV}$ is desirable, however only achievable in the presence of a magnetic field, where erratic currents are suppressed. A proper functionality of the BCML system, together with reasonable values of the measured MIB, is a requirement to allow the CMS Tracker to switch on the $\mathrm{HV}$, and is therefore a key ingredient in the CMS data taking.

\subsection{Beam timing and triggers}

Beam timing is checked before the LHC goes into collisions. The precision of the $\Delta T(\mathrm{~B} 1-\mathrm{B} 2)$ measurement in BPTX is about $50 \mathrm{ps}$, which corresponds to a precision on the position of the collision point of about $7.5 \mathrm{~mm}$. The number of bunches, paired and un-paired, can be cross-checked with the filling scheme. Signals from BPTX are used as technical triggers in CMS. These are: single bunch triggers, zero bias (AND), unpaired bunches (XOR), pre-collision (AND, 1 BX advanced),
(Fill 3679, Circulating Beams, 5 May 2015)

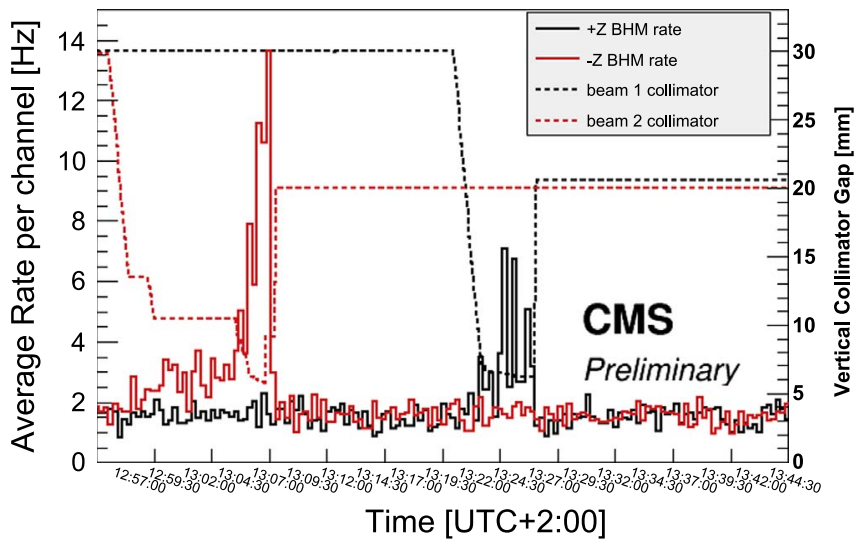

Fig. 9. Signals measured in BHM due to beam loss created in collimator scans together with respective collimator positions.

pre-train (1 BX before a train).

\section{Outlook for high luminosity (HL)-LHC}

For the phase-2 upgrade of CMS [14] the machine interface will be

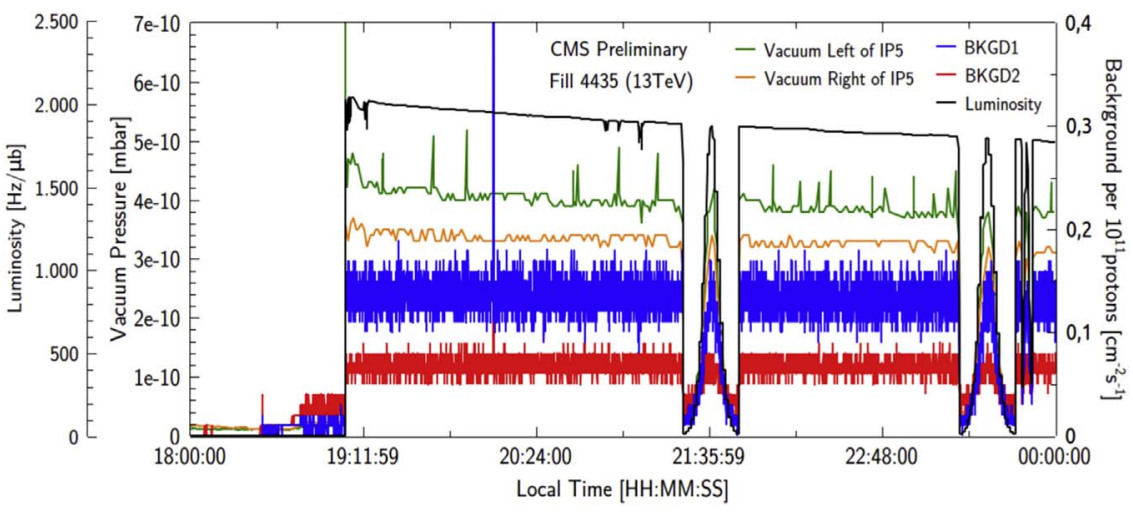

Fig. 8. Luminosity, MIB and vacuum close to CMS. 


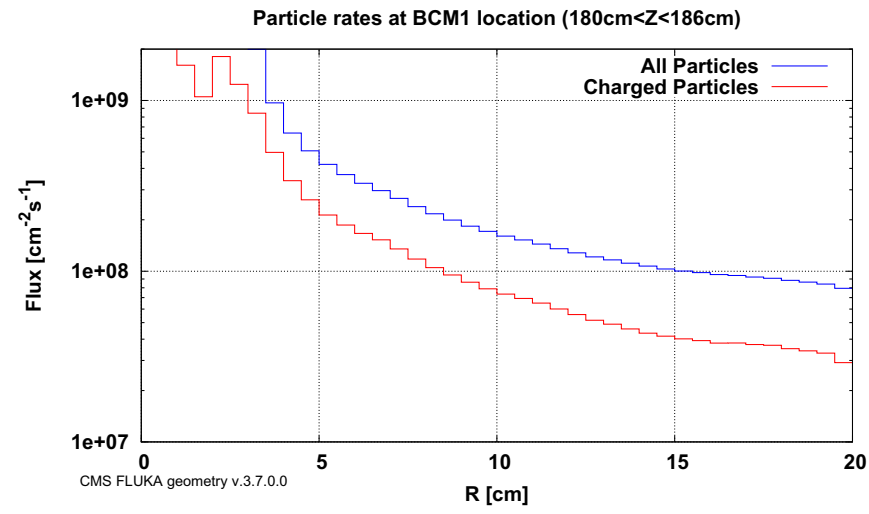

Fig. 10. FLUKA simulated particle rates at the BCM1/PLT location at HL-LHC conditions [14].

modified. The forward absorbers protecting CMS will be increased and the MIB rates will increase. The beam pipe radius in the forward region will be increased requiring a re-design of the BRIL systems there.

The CMS Tracker will be re-designed, requiring a modification of the BCM1 and PLT systems. All systems have to be designed to operate at a pileup of 150 and with a radiation hardness to survive an integrated luminosity of $3000 \mathrm{fb}^{-1}$. Especially luminometers require a high dynamic range and good linearity. The FLUKA simulated particle rates at the BCM1/PLT location at HL-LHC conditions is shown in Fig. 10. Specific modifications proposed to all beam instrumentation systems are:

- The current concept of the PLT is still a viable option for phase-2, however the CMS Pixel detector will be extended to $\eta=4$ and with it, more volume is needed. A luminosity system could be better integrated into the Pixel detector system by including a readout chain dedicated for luminosity measurement.

- A replacement for BCM1F has to be able to cope with increasing pileup. A higher granularity for high rate and similar total detector area for low rate measurements make single-channel readout increasingly difficult. A pixelated readout using a fast front-end chip that supports a readout frequency $>80 \mathrm{MHz}$ could be a solution.

- The BHM system is expected to perform well in HL-LHC conditions and does not have to be modified.
- The BCML system will be modified mechanically, and the detectors might get replaced. Possible alternatives to the diamond sensors are sapphire sensors [15] and small ionization chambers for the forward region where more space is available. The readout electronics will be the same as for the LHC BLM system.

- Similar to HFlumi, a calorimeter-based readout system could be included into the upgrades of any of the calorimeter systems. The performance of the phase- 2 detectors has to be studied.

\section{References}

[1] A. Dabrowski, et. al., The performance of the beam conditions and radiation monitoring system of CMS, in: Proceedings of the IEEE Nuclear Science Symposium and Medical Imaging Conference (NSS/MIC), 2011, pp. 489-495, 〈http://dx.doi.org/10.1109/NSSMIC.2011.6153979http://dx.doi.org/10.1109/ NSSMIC.2011.6153979).

[2] M. Guthoff, Design and experiences with the beam condition monitor as protection system in the CMS experiment of the LHC, in: Proceedings of the European Workshop on Beam Diagnostics and Instrumentation for Particle Accelerators, DIPAC'11, Hamburg, Germany, 2011.

[3] M. Guthoff, et al., The CMS/TOTEM FLUKA Nominal FLUKA Model, Version 1.0. 0.0, CERN-CMS-NOTE-2015-004.

[4] S. Mallows et al., Monte Carlo simulations of the radiation environment for the CMS experiment, http://dx.doi.org/10.1016/j.nima.2015.11.044.

[5] A. Ferrari, P.R. Sala, A. Fassò, J. Ranft, Fluka: a multi-particle transport code, CERN-2005-10, INFN/TC 05/11, SLAC-R-773, 2005.

[6] T.T. Böhlen, et al., The FLUKA code: developments and challenges for high energy and medical applications, Nucl. Data Sheets 120 (2014) 211-214.

[7] I. Azhgirey, et al., in: Proceedings of XVIII Workshop of the Charged Particle s Accelerators, Protvino, 2000.

[8] R. Bruce, et al., Sources of machine-induced background in the ATLAS and CMS detectors at the CERN Large Hadron Collider, Nucl. Instrum. Methods Phys. Res. A 729 (2013) 825.

[9] A. Kornmayer, The CMS pixel luminosity telescope, http://dx.doi.org/10.1016/j. nima.2015.09.104

[10] A. Zagozdzinska, The CMS fast beams condition monitor back-end electronics based on MicroTCA technology, in: IEEE NSS, 2015 in press, CMS CR-2015/315

[11] S. Orfanelli, et al., A novel beam halo monitor for the CMS experiment at the LHC, J. Instrum. 10 (2015) P11011.

[12] M. Guthoff, et al., Radiation damage in the diamond based beam condition monitors of the CMS experiment at the Large Hadron Collider (LHC) at CERN, Nucl. Instrum. Methods Phys. Res. A 730 (2013) 168-173. http://dx.doi.org/ 10.1016/j.nima.2013.05.041.

[13] The CMS Collaboration, CMS Luminosity Measurement for the 2015 Data Taking Period, CMS-PAS-LUM-15-001.

[14] J. Butler, D. Contardo, M. Klute, J. Mans, L. Silvestris, Technical Proposal for the Phase-II Upgrade of the CMS Detector, CERN-LHCC-2015-010, LHCC-P-008.

[15] O. Karacheban, et al., Investigation of a direction sensitive sapphire detector stack at the $5 \mathrm{GeV}$ electron beam at DESY-II, J. Instrum. 10 (2015) P08008. 